\section{Keratoconus and Acute Hydrops: Global Perspective}

Sir,

Whilst recently participating at an eye camp in Kenya, we came across a sad case of a 16-year-old girl, who presented with advanced keratoconus and acute hydrops (Figure 1). In addition to the physical and functional issues that she faced due to constant epiphora, she described social distress amongst her friends. Keratoconus is a progressive eye disease in which the configuration of the cornea changes. In the general population, the estimated prevalence of keratoconus is 1 per 2,000 people. 1 As the disease progresses, vision deteriorates, and corneal transplantation may be needed. Acutely, if Descemet's membrane ruptures, aqueous humour passes into the cornea and vision worsens (acute hydrops). This membrane breach can take up to 10 weeks to heal and patients can be left with scarring. Interestingly through the healing process, secondary to scarring and corneal flattening, visual acuity may improve.

In developed countries such as UK, where established and free healthcare via the National Health Service (NHS) is available, the vast majority of the population is managed and treated as needed. This is not the case for many parts of the developing world, where patients typically present late due to issues with both access to healthcare and healthcare literacy. Moreover, they may not receive optimal treatment due to cost burden.

Genetic and environmental factors appear to play a role in the complex and multifactorial etiology of keratoconus. ${ }^{2} \mathrm{~A}$ positive family history is also significant. In certain parts of the world such as Asia and Africa, consanguineous marriage is a social norm. Such practices are known to increase the risk for birth defects and the transmission of genetic conditions. ${ }^{3} \mathrm{~A}$ recent study by Jamali et al. found that in the pathogenesis of keratoconus, consanguineous marriage may play a role. 4 They also recommended that as part of screening programmes, in all healthcare systems, consanguineous marriages should not be overlooked. This is because research has shown that the risk of keratoconus may be 4 to 5 times higher in children whose parents are related compared to unrelated parents. ${ }^{5}$

Keratoconus is a global problem and consanguineous marriages are a major public health issue which increase the risk of keratoconus. In certain communities, a thorough family history is needed. Patient education

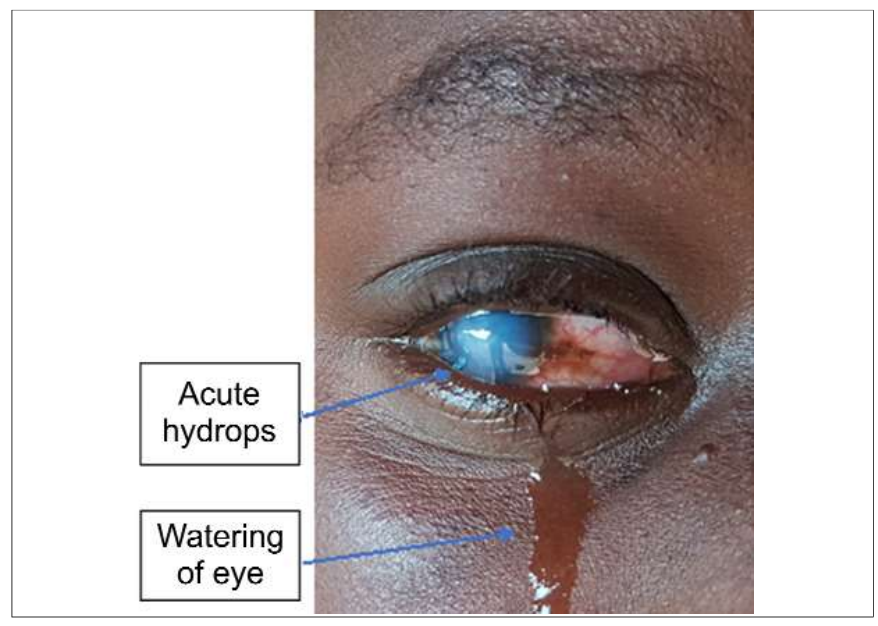

Figure 1: Acue coroneal hydrops.

pertaining to both keratoconus and consanguineous marriages would be beneficial. Better access to healthcare in addition to more affordable treatments would help in preventing patients becoming visually disabled.

\section{REFERENCES}

1. Ziaei $H$, Jafarinasab MR, Javadi MA, Karimian F, Poorsalman $H$, Mahdavi $\mathrm{M}$, et al. Epidemiology of keratoconus in an Iranian population. Cornea 2012; 31:1044-7

2. Akkaya S. Rate of parental consanguineous marriage among patients with visual impairments in Turkey. Med Hypothesis Discov Innov Ophthalmol 2016; 5:115-20.

3. Kumaramanickavel G, Joseph B, Vidhya A, Arokiasamy T, Shetty N. Consanguinity and ocular genetic diseases in South India: analysis of a five-year study. Community Genet 2002; 5:182-5.

4. Jamali $H$, Beigi V, Sadeghi-Sarvestani A. Consanguineous marriage as a risk factor for developing keratoconus. Med Hypothesis Discov Innov Ophthalmol 2018; 7:17-21.

5. Ansar M, Ramzan M, Pham TL. Localisation of a novel autosomal recessive non-syndromic hearing impairment locus (DFNB38) to 6q26-q27 in a consanguineous kindred from Pakistan. Hum Hered 2003; 55:71-4.

Shahzaib Rehan and Zartash Rehan

Department of Internal Medicine, University Hospital of Wales, Heath Park Way, Health Park, Cardiff, CF 14 XXW, Wales, UK

Correspondence: Dr. Shahzaib Rehan, Department of Internal Medicine, University Hospital of Wales, Heath Park Way,

Health Park, Cardiff, CF $144 X W$, Wales, UK

E-mail: shazmrehan@gmail.com

Received: June 09, 2018; Accepted: September 11, 2018 